\title{
SURFACE MORPHOLOGY OF COMETARY NUCLEI
}

\author{
D. MÖHLMANN AND E. KÜHRT \\ Institut für Kosmosforschung \\ Rudower Chaussee 5 \\ Berlin-Adlershof \\ $D D R-1199$
}

\begin{abstract}
The first in situ observations of a cometary nucleus by imaging techniques on board the VEGA and Giotto spacecraft in 1986 opened the possibility of directly studying the properties and structures of a cometary surface. This new information, combined with results from other experiments made by these spacecraft and with results from laboratory experiments and Earth-bound radar observations, gave a better basis for coming from speculation to more precise knowledge in describing properties of cometary surfaces and related activity. It is the aim of this paper to discuss the different views developed to understand properties of cometary surfaces and to propose a consistent model for understanding topological surface properties and related cometary activity. We accomplish this by extending former hypotheses and approaches and making them more precise in the light of the new and more complex data.
\end{abstract}

\section{Introduction}

During the last three decades, approaches to describing cometary nuclei and their surface properties were based upon Whipple's (1950) model of an icy conglomerate singlebody nucleus ("dirty snowball"). Using this guiding idea, the following pre-1986 aspects were worked out to describe surface structures and related processes of cometary nuclei:

- $\quad$ Formation of a dust surface layer ("crust"), modulating sublimation and outgassing. The crust structure is dependent on the effectiveness of sublimation and of gas and dust transport through this crust and on erosion at the crust (Whipple, 1950; Mendis and Brin, 1977; Brin and Mendis, 1979; Fanale and Salveil, 1984).

- The "friable sponge" model of an eroded surface, where greater grains break into smaller pieces (Horanyi et al., 1982).

- The "two-phase" surface of "active" sublimating regions and inactive areas of nonvolatiles and dust (Shulman, 1972; Whipple, 1978). 
- $\quad$ Repetitive local activity of discrete dust sources (Sekanina and Larson, 1984 and 1986).

In principle, the spacecraft observations of 1986 validated this basic approach of a crust covering the cometary surface. The new (post-1986) and more complex results will be discussed next.

\section{Summary of Spacecraft Imaging Results}

Surface features of Comet Halley are shown in Figure 1, which is a processed version of the original image that was taken, at a distance of $8,000 \mathrm{~km}$ from the nucleus, by VEGA 2 about $2 \mathrm{~s}$ before closest encounter. This "full comet" image was taken at a Sun-comet-spacecraft angle of $28.4^{\circ}$. In analogy to "full Moon" images with their weak contrast, this image displays only some crater-like features, a global deformation ("central depression"), and large, relatively dark regions with embedded bright spots - indicating local activity-and coherent "lineamental" zones of moderate activity. In principle, the same features can be seen, with better resolution, but from a smaller illuminated part of the surface, in Giotto's more contrast-rich images, which are analogous to "young Moon" images. Figure 2 gives a schematic sketch of Comet Halley's surface features as seen from the two spacecraft. It is interesting to note that the spacecraft looked onto nearly opposite hemispheres of the nucleus. Obviously, the results of imaging experiments on board both VEGA and Giotto gave comparable information about cometary surface topography. These common results are that surface properties, topography, and morphology of Comet Halley's nucleus can be characterized by (Keller et al., 1986; Reitsema et al., 1986; Möhlmann and Kührt, 1989; Curdt and Keller, 1989):

- An irregular shape and surface.

- A "hilly" surface with "crater-like" features, mountains, and slopes with common scale-lengths between about a hundred meters and a few kilometers (well above the maximum resolution of about $100 \mathrm{~m}$ ).

- $\quad$ A large-scale (global) "central depression."

- The existence of grains in the neighborhood of the cometary nucleus.

- A remarkably dark surface with $3 \%$ to $4 \%$ albedo.

- Inactivity (i.e., strongly reduced outgassing) on about $90 \%$ of the cometary surface. This indicates that sublimation and gas transport are effectively reduced by a crust covering most of Comet Halley's surface.

- The restriction of cometary activity to point-like local "sources of activity," partially with repetitive activity.

- Jets, which are coming from square-kilometer-sized (or smaller) sources and are collimated (focused). The jets also show fine structures of dust filaments (with scales on the order of some hundred meters).

- The appearance of "moderate" cometary activity in connected ("coherent") lineamental zones. It is possible that this impression is the result of an unresolved distribution of weaker sources. 


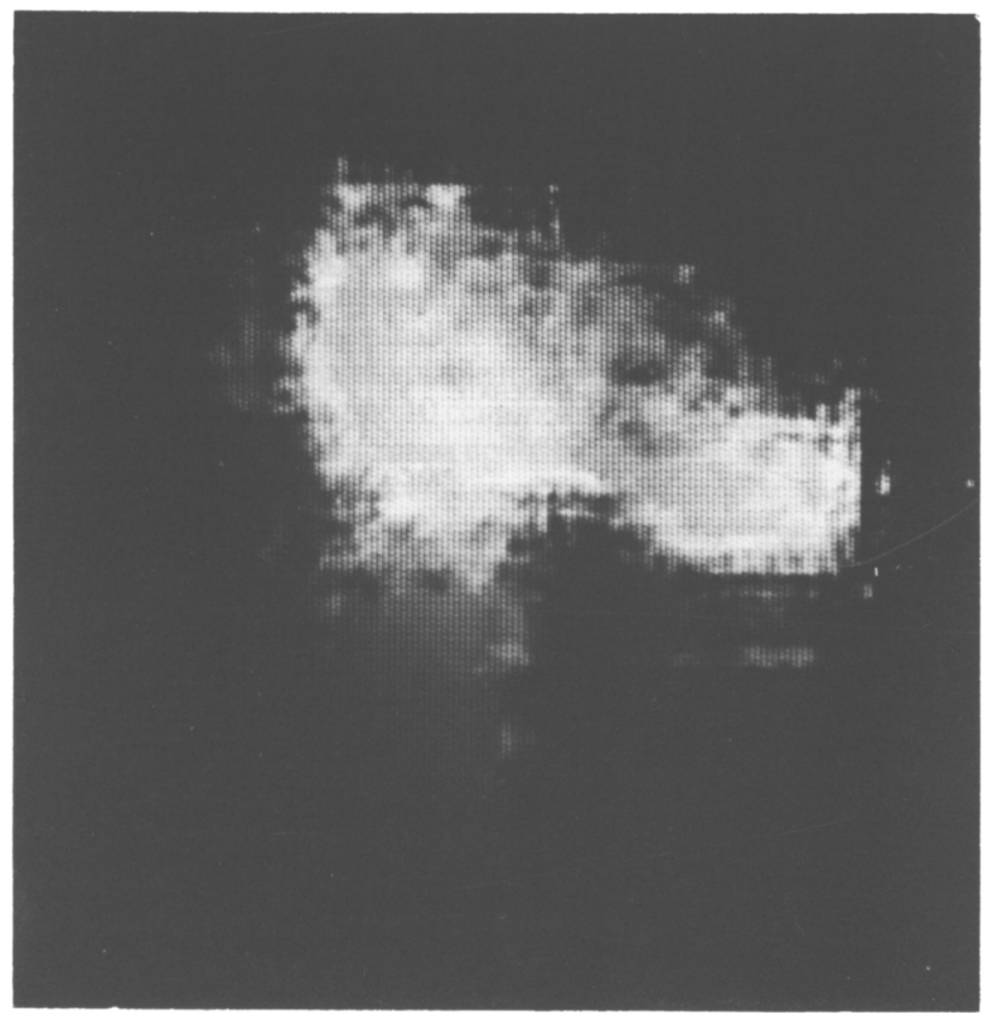

Figure 1. Processed image of the nucleus of comet Halley, as seen from VEGA 2 at a distance of about $8,000 \mathrm{~km}$.

A further remarkable result came from Earth-bound radar observations of comets (including P/Halley): Cometary nuclei are surrounded by a cloud of (orbiting) centimetersized particles (Campbell et al., 1989; Harmon et al., 1989).

These observations provide a serious indication of the existence of compact aggregations of solid grains of a nonvolatile nature at (and in) cometary nuclei. Up to now, the aggregates' existence was postulated only from observations of meteor streams connected with comets. Furthermore, and in possible correlation with the now-accepted existence of centimeter-sized aggregations, these radar observations have shown that the "surface roughness scale" of cometary nuclei is on the order of centimeters. Consequently, cometary surfaces seem to be covered in inactive regions by an upper crust or "mantle" of aggregate depositions. 
VEGA HEMISPHERE

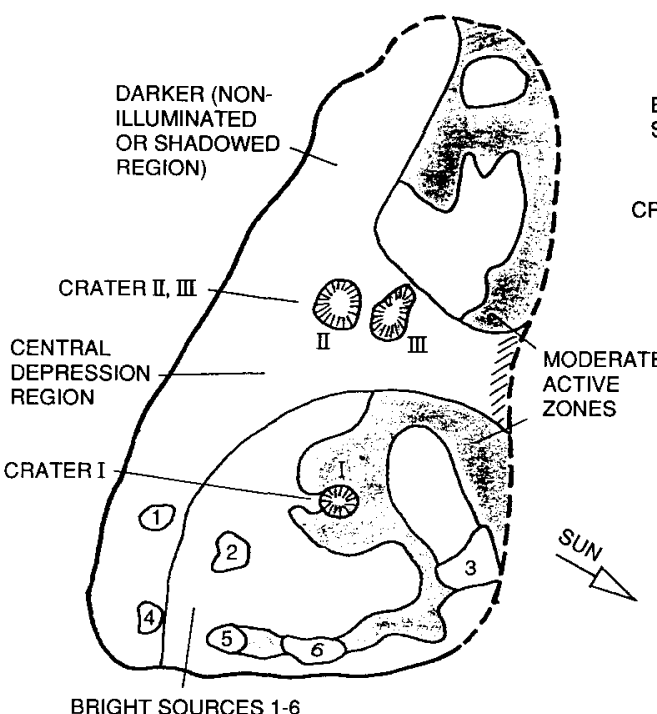

GIOTTO HEMISPHERE

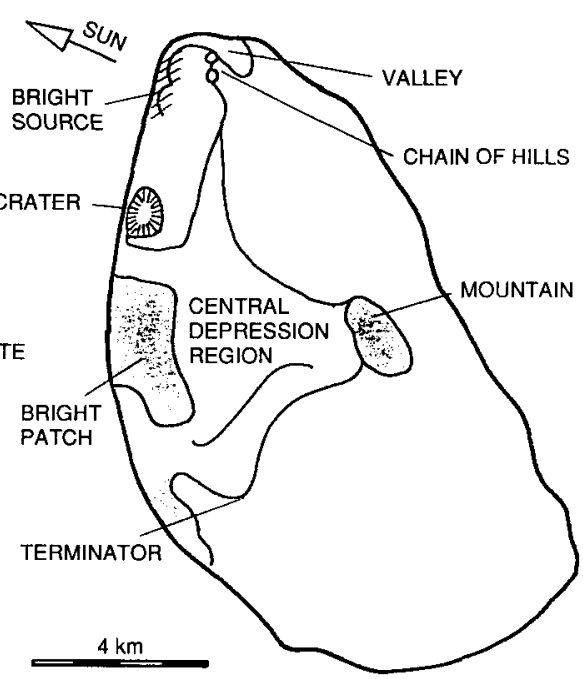

Figure 2. Schematic sketch of surface features of comet Halley, derived from VEGA and Giotto imaging results.

\section{Model of Cometary Activity}

The main task in understanding structures and properties of cometary surfaces seems to be to bring the following key aspects together into a consistent picture:

- The existence of a crust, which restricts sublimation, gas and dust transport, and related cometary activity.

- The existence of a cloud of centimeter-sized particles (grain aggregations) surrounding cometary nuclei.

- Surface roughness on the order of centimeters.

- Local point-like and repetitive "sources of activity."

- Typical horizontal and vertical surface scales of between some hundred meters and a few kilometers. 
The first successful attempt to bring these pieces together was made by Wallis and Wickramasinghe (1987) by combining Shulman's (1972) "two-phase" concept, the "stone hail" of Kührt et al. (1986) - and its tendency to make inactive areas still more dormantand the idea that "crater-like" features or hollows could be sublimation-generated. The more general underlying concept, as will be discussed now, is that of a cometary surface changed steadily by local regions of activity, "moving at sublimation fronts" over the surface. Of course, this model of surface evolution has to be related to the internal structure of a cometary nucleus. But as a first approach, taking into account the great uncertainties in modeling internal structures of cometary nuclei (Houpis, 1987), only one assumption about cometary nuclei's internal properties shall be used, namely that particles ("grain aggregates") exist embedded in a matrix of volatile matter. These particles can range in size between micrometers (dust) and some 10 centimeters. Even greater sizes are possible. Using the fact that particles with diameters up to the order of some 10 centimeters (depending on the heliocentric distance, the cometocentric latitude, and the season) can be lifted off the nucleus and accelerated by the gas stream of the sublimating volatiles into escaping, orbiting, or ballistic motions, it becomes clear that cometary nuclei must be

- Surrounded by an apparent cloud of centimeter-sized particles (Kührt et al., 1986; Banaszkiewicz et al., 1989), and

- Covered by a "mantle" of a mixture of never-lifted large particles, particles that have fallen back ("stone hail"), and those smaller grains that are hindered in their motion through the surface by larger particles (e.g., by sticking).

Consequently, those parts of the cometary surface that are covered by this "mantle" should be inactive, because the volatiles are thermally insulated. Since grains with ballistic trajectories can fall back only onto less active regions, passive surface areas tend to become still more dormant. The reactivation of such inactive areas is made possible only by the breaking of the mantle, e.g., by meteoritic impacts, thermal stresses (Tauber and Kührt, 1987), or eruptive events caused by inner heat sources (Prialnik and Bar-Nun, 1988) or chemical inhomogeneities, or the "undermining" by "moving sublimation fronts (slopes)."

Obviously, a stably deposited pebble-mantle cannot be formed on strongly inclined surface features such as slopes, crater-like structures, mountains, trenches, and crevasses, because particles can roll down to the bottom of such structures if their inclination is sufficiently great. This effect was also demonstrated by the KOSI experiments (Grün et al., 1989). Therefore, the strongly inclined parts of the surface are potential sources (loci) of cometary activity. It is interesting to note that in concave surface features, sublimation will be enhanced due to the thermal focusing effect. The net result will be for such features to accentuate, rather than to diminish, in magnitude (Colwell and Jakosky, 1987). Two connected results are that such structures grow laterally, while the ground is filled by unlifted larger particles.

Furthermore, it must be mentioned that these inclined surfaces may make an essential contribution to the observed focusing of jets and their fine structure.

The proposed surface-morphology-related model of cometary activity can explain the above-mentioned observed cometary properties (e.g., surface topography, local roughness, local activity, and orbiting particle clouds), without hypothetical assumptions about internal structures. Obviously, the surface-related activity steadily (but slowly) changes the 
surface morphology of a cometary nucleus, steadily creating a new surface topography.

Within the frame of this approach, the maximum surface scales $S$ should be on the order of $S=N^{*} D$, where $N$ is the number of apparitions (in one type of orbit) and $D$ is the typical "depth" of sublimation-caused surface reduction over the period of one apparition. For $\mathrm{P} / \mathrm{Halley}$, we have (as a first estimation) an $\mathrm{N}$ of perhaps a hundred to a thousand and a D of a few meters. The observed scales reflect these estimated values for $\mathrm{P} / \mathrm{Halley}$ very well indeed.

Information about internal structures can be derived only from large-scale features, such as the central depression and coherent zones of moderate activity. These features favor models of a "block structure" of cometary nuclei, made of "building blocks" (Weissman, 1986; Möhlmann et al., 1986). Such models are supported by the observations of strong outbursts and splittings. But to derive this from the VEGA and Giotto images is a possible but not exclusively conclusive interpretation. So this problem remains open.

\section{Conclusions}

Ground-based observations and the 1986 Halley missions gave a new basis of data and facts to describe the surface properties of cometary nuclei, including the phenomena of cometary activity. Using the proposed model of "active slopes," this paper has shown that cometary activity seems to be related to the surface morphology in such a way that large passive areas are covered by a thermally insulating "mantle" of a "processed" mixture of larger particles, dust, and condensates, while cometary activity is connected with "active slopes," in this way steadily creating new surfaces by the lateral growth of the slopes. The heating of the slopes will tend also "to form overhanging rims, presumably crumbling or breaking away in chunks as further sublimation undermines" them (Wallis and Wickramasinghe, 1987).

A future task is to check this model by direct imaging of active regions and by verifying that the sources of activity are inclined slopes. Unfortunately, the imaging results of VEGA and Giotto are not sufficient to prove or disprove this model directly.

\section{Acknowledgment}

The authors wish to thank Dr. Ray L. Newburn, Jr., for patience and careful help in preparing this paper. We have to thank also Dr. Rickman for helpful critical comments.

\section{References}

Banaszkiewicz, M., et al. (1989). "Dynamics of large dust grains around Halley's comet," this volume.

Brin, G.D., and Mendis, D.A. (1979). Astrophys. J. 229, 402.

Campbell, D.B., et al. (1989). "Radar observations of comet Halley," Ap. J. 338, 1094 1105 . 
Colwell, J.E., and Jakosky, B.M. (1987). "Evolution of topography on a comet," Icarus $72,128-134$.

Curdt, W., and Keller, H.U. (1989). "Large dust particle fluence along the Giotto trajectory," poster presented at the Bamberg meeting.

Fanale, F.P., and Salveil, J.R. (1984), Icarus 72, 123.

Grün, E., et al. (1989). "Modifications of comet materials by the sublimation process: Results from simulation experiments," Proceedings of Workshop "Analysis of Returned Comet Nucleus Samples."

Harmon, J.K., et al. (1989). "Radar observations of comet IRAS-Araki-Alcock 1983d," Ap. J. 338, 1071-1093.

Horanyi, M., and Kecskemety, K. (1982). In Cometary Exploration, Hung. Acad. Sci., p. 59.

Houpis, H.L.F. (1987). "Models of cometary nuclei," in Comet Halley 1986Worldwide Investigations, Results and Interpretations, A. Horwood, England.

Keller, H.U., et al. (1986). "Observations by the Halley multi-colour camera," ESA SP-250, Vol. II, 347-350.

Kührt, E., et al. (1986). "Thermal stresses and dust dynamics on comets," ESA SP-250, Vol. II, 385-388.

Mendis, D.A., and Brin, G.D. (1977). Moon and Planets 17, 359.

Möhlmann, D., et al. (1986). "Physical properties of P/Halley - derived from VEGAimages," ESA SP-250, Vol. II, 339-340, and "Image processing of VEGA-TV observations," ESA SP-250, Vol. III, 313-315.

Möhlmann, D., and Kührt, E. (1989). "Comet nucleus models," Adv. Space Res. 9 (3), (3)17-(3) 23 .

Prialnik, D., and Bar-Nun, A. (1988). "The formation of a permanent dust mantle and its effect on cometary activity," Icarus 69, 83-90.

Reitsema, H.J., et al. (1986). "Nucleus morphology of comet Halley," ESA SP-250, Vol. II, 351-354.

Sekanina, Z., and Larson, S.M. (1984). Astron. J. 89, 1408 and 1446, and (1986), Astron. J. 92, 462.

Shulman, E.M. (1972). In The Motion, Evolution of Orbits and Origin of Comets, D. Reidel, Dordrecht, p. 265.

Wallis, M.K., and Wickramasinghe, N.C. (1987). "Crustal models of the evolving comet nucleus," ESA SP-278, 495-499.

Weissman, P.R. (1986). "How pristine are cometary nuclei?", ESA SP-249, 15-25.

Whipple, F.L. (1950), Astron. J. 111, 375.

Whipple, F.L. (1978), Moon and Planets 18, 343. 